\title{
Neutrophil-Lymphocyte Ratio as A Complementary Biomarker of Periprosthetic Joint Infection: A Retrospective Review
}

\section{Yongyu Ye}

First Affiliated Hospital of Sun Yat-sen University

Minghui Gu

First Affiliated Hospital of Sun Yat-sen University

\section{Weishen Chen}

First Affiliated Hospital of Sun Yat-sen University

\section{Guoyan Xian}

First Affiliated Hospital of Sun Yat-sen University

\section{Linli Zheng}

First Affiliated Hospital of Sun Yat-sen University

\section{Baiqi Pan}

First Affiliated Hospital of Sun Yat-sen University

\section{Ziji Zhang ( $\sim$ zhangziji1984@163.com )}

First Affiliated Hospital of Sun Yat-sen University

Puyi Sheng ( $\nabla$ shengpuyi@hotmail.com )

First Affiliated Hospital of Sun Yat-sen University https://orcid.org/0000-0002-5898-2161

\section{Research article}

Keywords: periprosthetic joint infection, neutrophil-lymphocyte ratio, diagnosis

Posted Date: May 12th, 2020

DOI: https://doi.org/10.21203/rs.3.rs-26625/v1

License: (1) (1) This work is licensed under a Creative Commons Attribution 4.0 International License. Read Full License 


\section{Abstract}

Background: Diagnosing a periprosthetic joint infection (PJI) is challenging. No single biomarker can accurately recognize PJI preoperatively in a timely manner. Therefore, the aim of the present study was to investigate the usefulness of the neutrophil-lymphocyte ratio (NLR) in aiding the diagnosis of PJI.

Methods: We retrospectively evaluated the medical records of 109 patients who had undergone revision arthroplasty (77 with aseptic mechanic failure and 32 with PJI) from July 2011 to November 2018. Bivariate analysis followed by multivariate logistic regression was applied to compare NLR between two groups. The receiver operating characteristic (ROC) curve was used to assess the diagnostic performance of NLR alone and in combination with C-reactive protein (CRP) and the erythrocyte sedimentation ratio (ESR).

Results: NLR was significantly higher in patients with PJI than in patients of the aseptic revision group $(3.12 \pm 2.45$ vs. $2.10 \pm 1.03, p<0.05)$. ROC curve analysis revealed that NLR had a sensitivity of $56.25 \%$ and a specificity of $80.52 \%$ with an optimal threshold of 2.52 . The diagnostic performance of NLR alone was inferior to that of CRP and ESR as demonstrated by the area under curve, sensitivity, and diagnostic odds ratio. When used in combination with $\mathrm{CRP}$, the diagnostic performance was nearly equal to the combined performance of CRP and ESR or that of all three biomarkers $(p>0.05)$.

Conclusions: NLR is associated with PJI and could be regarded as a complementary marker, which in combination with CRP could improve the diagnostic performance for PJI.

\section{Background}

Although significant progress has been made in total joint arthroplasty (TJA), periprosthetic joint infection (PJI) remains one of the most disastrous postoperative complications. It leads to a lengthy hospital stay, places a huge economic burden, and increases morbidity and mortality rates [1-3]. It has been claimed that approximately 1 to $2.5 \%$ of orthopedic patients undergoing primary TJA will develop PJIs [3-5]. However, the timely and accurate diagnosis of PJI continuous to be a challenge for surgeons. Despite the suggestion of several biomarkers, including the C-reactive protein (CRP) and the erythrocyte sedimentation rate (ESR), for the diagnosis of PJI, as recommended by the Musculoskeletal Infection Society (MSIS) and the 2018 International Consensus Meeting, there are still no well-documented and reliable markers that could help surgeons obtain the right and timely preoperative diagnosis $[1,6]$. Both the CRP and ESR could be within the normal range in some patients with PJI, particularly in those with chronic and low virulence infections [7]. Additionally, the guideline requires the performance of an invasive procedure or intraoperative exploration. Therefore, the requirement for easily accessible, noninvasive, and cost-effective markers to assist with the timely and correct diagnosis of PJI is warranted.

The neutrophil-lymphocyte ratio (NLR), an easily accessible, cost-effective, and reproducible marker, is calculated from the complete blood count (CBC), which is recognized as a well-established marker of the 
systemic inflammatory response [8]. The NLR has been demonstrated to be a crucial marker for solid tumors and systemic rheumatic diseases, such as rheumatoid arthritis [9-11]. Recently, studies have shown that the NLR was related to the postoperative pain and surgical site infection in orthopedic surgery and might be a predictive marker for deep vein thrombosis after total knee arthroplasty [12-14]. Additionally, Golge reported that NLR might be useful in the diagnosis of PJI, increasing the accuracy of the diagnosis when combined with CRP and ESR [15]. However, to the best of our knowledge, no solid evidence was found regarding the diagnostic value of NLR in predicting PJI.

Hence, the aim of the present study was to investigate the diagnostic performance of NLR in predicting PJI in comparison with that of CRP and ESR in patients with total hip or knee arthroplasties and explore the complementary value of NLR in the diagnosis of PJI when combined with CRP and ESR.

\section{Material And Methods}

\section{Study design and selection criteria}

Following approval by our institutional review board, we conducted this single-center, retrospective review of patients who had undergone revision total hip or knee arthroplasties from July 2011 to November 2018 in our hospital. Initially, a total of 173 patients having undergone revision arthroplasty were enrolled. Respectively, 64 patients were excluded from our study based on the following exclusion criteria: 1) patients with underlying inflammatory conditions, e.g. for rheumatoid arthritis $(n=4)$ and ankylosing spondyloarthritis $(n=3) ; 2)$ patients experiencing other infectious conditions, such as tuberculosis $(n=3)$ and carbuncle $(n=2) ; 3$ ) patients undergone revision surgery because of acute PJI (occurred < 3 mo from the primary surgery, $n=1)$, dislocation $(n=16)$, periprosthetic fracture (most of them were due to violence, $n=5)$, breakage of prosthesis $(n=1)$ and surgical site infection $(n=10) ; 4)$ patients with severe dysfunction of kidney $(n=2) ; 5)$ lack of preoperatively recorded $\operatorname{CRP}(n=9)$ or $\operatorname{ESR}(n=8)$ values. Finally, the medical records of 77 patients with aseptic mechanic failure (aseptic loosening and wear, malalignment, instability, or other unexplained pain) and 32 patients with septic revision were analyzed in the study. The requirement for informed consent was waived due to the retrospective design of our study.

\section{Diagnostic criteria and protocol}

The diagnosis of PJI in our institution was defined by the MSIS criteria $[1,6]$. All revision patients with the suspicion of PJI followed the algorithm of assessment in our joint surgery department, modified from MSIS. Routine blood tests would be ordered for all patients preoperatively. After the comprehensive evaluation of patients, those for which clinical suspicion of PJI existed, significantly elevated serological infection-related markers, or positive imaging findings, were subjected to preoperatively aspiration and samples were delivered to the lab for further assessment of bacterial culture. Moreover, analysis of the synovial fluid (color, clarity, Rivalta test, white blood cell count, polymorphonuclear differential, and gram stain as needed) was also required. Preoperative antibiotics were not routinely prescribed unless we had such instructions based on the obtained results from the sensitivity test of bacterial culture. Additionally, at least 2 cultures were obtained from different sites before the irrigation of iodine fluid for at least 3 
times intraoperatively. Recently, 1 or 2 pathologic periprosthetic tissues were biopsied during surgery and sent for histologic analysis. Second-generation cephalosporin was routinely initiated during and continued after surgery. However, in case doctors had a positive result from the sensitivity test of bacterial culture, sensitive antibiotics would be prescribed. All patients received prophylactic heparin or other antithrombotic therapy to prevent deep vein thrombosis within $24 \mathrm{~h}$ postoperatively and for continuous $28 \mathrm{~d}$. Last, $\mathrm{CBC}$ and infection-related markers were regularly reexamined to monitor their fluctuations.

\section{Data review}

A detailed evaluation of medical records of all revision patients was carried out to review the demographic information and medical history. Baseline information included age, sex, smoking, alcohol, operative joint, admission, and discharge date. Comorbidities concerning rheumatoid arthritis, ankylosing spondylitis, diabetes mellitus, hypertension, and dysfunction of kidney or liver were considered. Operative details, including date of surgery, start time, end time, duration of surgery, and type of anesthesia were recorded. The management of revision patients, especially the use of antibiotics, was reviewed. Besides, in order to decrease the confounding effect, the regional infection (surgical site infection) and infections in other regions of the body (for example, tuberculosis and carbuncle) were also carefully documented. Last but not least, the reasons and diagnoses for readmission, including PJI, periprosthetic fracture, dislocation, breakage of prostheses, surgical site infection, and aseptic mechanic failure (aseptic loosening and wear, malalignment, instability, or other unexplained pain) were extracted from their electronic records.

\section{Laboratory evaluation}

Routine blood samples, such as CBC, liver enzyme and marker related to kidney function, were drawn in the morning preoperatively after patients underwent nighttime fasting for a minimum of $7 \mathrm{~h}$. Samples for detection of CRP and ESR were also collected in revision patients. Samples were sent to the medical laboratory in our hospital as soon as possible according to the accepted standards and controls. Neutrophils and lymphocytes were extracted from CBC retrospectively. The NLR was calculated as the proportion of the absolute count of neutrophils to the absolute count of lymphocytes.

\section{Statistical Analysis}

Mean and standard deviation (SD) were used to describe the quantitative data, while the number and percentage were calculated for categorical variables. An independent $t$ test was performed to detect the differences in age between groups. The categorical variables of demographic features were calculated with the use of a chi-square test. To evaluate the differences of each serologic biomarker between aseptic and septic groups, we used a Mann-Whitney $U$ test for comparisons. To decrease the effect of confounding factors, the adjusted $p$ value was assessed by the multivariate logistic regression (forward likelihood ratio method) regarding age, sex, operative joint, diabetes mellitus, and hypertension. The above analyses were performed using the Statistical Package for the Social Sciences (SPSS) version 22 (IBM Corporation, Armonk, NY, USA). The MedCalc Software (version 19.0.7, Ostend, Belgium) was 
employed to analyze the receiver operating characteristic (ROC) curves. The area under curve (AUC), sensitivity, specificity, positive predictive value (PPV), negative predictive value (NPV), positive likelihood ratio (+ LR), negative likelihood ratio (-LR), accuracy and diagnostic odds ratio (DOR) were used to investigate the diagnostic performance of NLR, CRP, ESR or combination of biomarkers. The Youden index was applied to determine the optimal threshold for the diagnosis of PJI. Variables with a $p$ value of $<0.05$ were considered statistically significant.

\section{Results}

\section{Demographic features of patients undergone revision surgery}

Finally, the medical records of 109 patients involved in our study, including 77 patients with aseptic mechanical failure and 32 patients with PJI, were assessed. The demographic features of both groups are shown in Table 1. Among patients with PJI, the average age was $66 \mathrm{y}$, with $40.64 \%$ being male. Noted, $15.6 \%$ of patients were reported to also feature diabetes mellitus. The baseline characteristics of age, sex ratio, and status of diabetes mellitus between the 2 groups did not exhibit significant differences $(p=$ $0.188,0.735$, and 0.576 , respectively). The rate of patients presented with hip issues in the aseptic revision group was significantly higher than that in the PJI group ( 88.3 vs. $59.4 \%, p=0.001)$. Additionally, the 2 groups did differ regarding the status of blood pressure, with the PJI group suffering more from hypertension ( 24.7 vs. $46.9 \%, p=0.023$ ). 
Table 1

Demographic Features of Patients Underwent Revision Surgery

\begin{tabular}{|c|c|c|c|}
\hline Group and Variable & Aseptic Revision & PJI & P Value \\
\hline No. of patients & 77 & 32 & \\
\hline Age* & $62.53 \pm 13.27$ & $66.03 \pm 10.57$ & $0.188^{a}$ \\
\hline Sext & & & $0.735^{b}$ \\
\hline Female & $43(55.8 \%)$ & $19(59.4 \%)$ & \\
\hline Male & $34(44.2 \%)$ & $13(40.64 \%)$ & \\
\hline Joint† & & & $0.001^{b}$ \\
\hline Hip & 68 (88.3\%) & $19(59.4 \%)$ & \\
\hline Knee & $9(11.7 \%)$ & $13(40.64 \%)$ & \\
\hline Diabetes Mellitust & & & $0.576^{b}$ \\
\hline Diabetic & $9(11.7 \%)$ & $5(15.6 \%)$ & \\
\hline Non-diabetic & 68 (88.3\%) & 27 (84.4\%) & \\
\hline Blood pressuret & & & $0.023^{b}$ \\
\hline Hypertension & $19(24.7 \%)$ & $15(46.9 \%)$ & \\
\hline Non-hypertension & $58(75.3 \%)$ & $17(53.1 \%)$ & \\
\hline \multicolumn{4}{|c|}{$\begin{array}{l}\text { *Data are presented as the mean } \pm \text { standard deviation. }+ \text { Data are presented as the number } \\
\text { (percentage) of patients. a } P \text { value was calculated by the independent } T \text { test. } \mathrm{b} P \text { value was calculated } \\
\text { by the chi-square test. } \mathrm{P}<0.05 \text { indicates a significant difference between groups. PJI, periprosthetic } \\
\text { joint infection. }\end{array}$} \\
\hline
\end{tabular}

\section{Comparisons of serum biomarkers between patients in aseptic and septic groups}

The serum neutrophil, lymphocyte, NLR, CRP, and ESR biomarkers were compared between the 2 groups. Except for lymphocytes, significant differences were noted between the aseptic and septic groups, as shown in Table 2 and Fig. 1. In patients of the septic group, the mean value of the count of neutrophils $\left(10^{\wedge} 9 / \mathrm{L}\right)$ was reported to be 4.97 , which was higher than that in patients of the aseptic group exhibiting a mean value of $3.66(p<0.001$, adjusted $p=0.001)$. The NLR for patients with previous diagnosis of PJI was 3.12 compared with 2.10 among patients in the aseptic revision group $(p=0.009$, adjusted $p=$ 0.024). The mean value of CRP in the PJI group was $25.08 \mathrm{mg} / \mathrm{L}$, which was significantly higher than that of the aseptic revision group with a mean value of $5.66 \mathrm{mg} / \mathrm{L}(p<0.001$, adjusted $p=0.002)$. Similarly, the 
level of ESR in patients with PJI was significantly different in comparison with that in patients of the aseptic revision group (53.88 vs. $28.42 \mathrm{~mm} / \mathrm{h}, \mathrm{p}$ and adjusted $\mathrm{p}<0.001)$.

Table 2

Comparison of Biomarkers Between the Aseptic and Septic Revision

\begin{tabular}{|lllll|}
\hline Biomarker & Aseptic Revision* & PJI* & P Value & Adjusted P Value \\
\hline NEUT (10^9/L) & $3.66 \pm 1.24$ & $4.97 \pm 2.00$ & $<0.001$ & 0.001 \\
\hline LYM $\left(10^{\wedge} 9 / \mathrm{L}\right)$ & $1.86 \pm 0.60$ & $1.96 \pm 0.86$ & 0.984 & 0.678 \\
\hline NLR & $2.10 \pm 1.03$ & $3.12 \pm 2.45$ & 0.009 & 0.024 \\
\hline CRP $(\mathrm{mg} / \mathrm{L})$ & $5.66 \pm 9.83$ & $25.08 \pm 34.69$ & $<0.001$ & 0.002 \\
\hline ESR $(\mathrm{mm} / \mathrm{h})$ & $28.42 \pm 21.10$ & $53.88 \pm 31.74$ & $<0.001$ & $<0.001$ \\
\hline $\begin{array}{l}\text { *Data are presented as the mean } \pm \text { standard deviation. P value was calculated by the Mann-Whitney U } \\
\text { test. Adjusted P value was assessed by the multivariate logistic regression (forward likelihood ratio }\end{array}$ \\
$\begin{array}{l}\text { method) regarding age, sex, joint, diabetes mellitus, and hypertension. P< } 0.05 \text { indicates a significant } \\
\text { difference between groups. PJl, periprosthetic joint infection; NEUT, neutrophil; LYM, lymphocyte; NLR, } \\
\text { neutrophil-lymphocyte ratio; CRP, C-reactive protein; ESR, erythrocyte sedimentation rate. }\end{array}$ \\
\hline
\end{tabular}

\section{The performance of NLR, CRP, and ESR in detecting PJI demonstrated by ROC curves}

Consecutively, ROC curves were used to depict the discriminatory performance of NLR, CRP, and ESR between the aseptic mechanic failure group and the PJI group. The respective AUC values for NLR, CRP, and ESR were demonstrated to be 0.658 (95\% confidence interval $0.561-0.746, p=0.009), 0.797(95 \%$ confidence interval $0.710-0.868, p<0.001$ ), and 0.747 (95\% confidence interval $0.654-0.825, p<0.001$ ). The sensitivity, specificity, PPV, and NPV for NLR in detecting PJI was 56.25, 80.52, 54.5, and 81.6\% respectively, with an optimal threshold $>2.52$. Moreover, its positive and negative likelihood ratio (LR) was 2.89 and 0.54 , with a $73.39 \%$ accuracy. The DOR of NLR was shown to be 5.31 . The optimal threshold with regards to CRP and ESR was evaluated to be $7.00 \mathrm{mg} / \mathrm{L}$ (sensitivity: $62.50 \%$, specificity: $83.12 \%$, PPV: $60.6 \%$, NPV: $84.2 \%$, +LR: 3.70, -LR: 0.45, accuracy: 77.07, and DOR: 8.21 ) and $43 \mathrm{~mm} / \mathrm{h}$ (sensitivity: 59.38\%, specificity: $80.52 \%$, PPV: 55.9\%, NPV: $82.7 \%$, +LR: 3.05, -LR: 0.50, accuracy: 74.31, and DOR: 6.04), respectively. The diagnostic performance of NLR, demonstrated by analysis of the ROC curve, was therefore shown to be inferior to that of CRP and ESR (Table 3, Fig. 2A). Additionally, we further evaluated the combined diagnostic value of NLR, CRP, and ESR for predicting PJI. The resulting AUC for the combination of NLR with CRP or ESR was shown to be 0.781 (95\% confidence interval $0.692-0.855, p<$ 0.001 ) and 0.741 (95\% confidence interval 0.648-0.820, $p<0.001$ ), respectively. Compared with just using CRP or ESR, the specificity (89.61 and 89.61\%), PPV (69.2 and 68.0\%), accuracy (79.82 and $78.90 \%$ ), and DOR (11.09 and 9.78) for the combination were greater, hence improving the diagnostic performance in predicting PJI. The diagnostic value of combining NLR and CRP was exhibited to be nearly equal to the conjunction of CRP and ESR or all 3 biomarkers together $(p>0.05)$. Moreover, no 
difference was demonstrated in diagnostic performance between combining 2 and 3 biomarkers $(p>$ 0.05) (Table 4, Table 5, Fig. 2B).

Table 3

Performance of NLR and CRP in the Diagnosis of PJI

\begin{tabular}{|llll|}
\hline & NLR & CRP & ESR \\
\hline AUC & 0.658 & 0.797 & 0.747 \\
\hline $95 \%$ Cl & $0.561-0.746$ & $0.710-0.868$ & $0.654-0.825$ \\
\hline Standard error & 0.061 & 0.047 & 0.052 \\
\hline Z-statistic & 2.608 & 6.323 & 4.714 \\
\hline P Value & 0.009 & $<0.001$ & $<0.001$ \\
\hline Threshold & 2.52 & 7.00 & 43 \\
\hline Sensitivity (\%) & 56.25 & 62.50 & 59.38 \\
\hline Specificity (\%) & 80.52 & 83.12 & 80.52 \\
\hline PPV (\%) & 54.5 & 60.6 & 55.9 \\
\hline NPV (\%) & 81.6 & 84.2 & 82.7 \\
\hline +LR & 2.89 & 3.70 & 3.05 \\
\hline -LR & 0.54 & 0.45 & 0.50 \\
\hline Accuracy (\%) & 73.39 & 77.07 & 74.31 \\
\hline DOR & 5.31 & 8.21 & 6.04 \\
\hline $\begin{array}{l}\text { PJI, periprosthetic joint infection; NLR, neutrophil-lymphocyte ratio; CRP, C-reactive protein; ESR, } \\
\text { erythrocyte sedimentation rate; AUC, area under curve; Cl, confidence interval; PPV, positive predictive } \\
\text { value; NPV, negative predictive value; +LR, positive likelihood ratio; -LR, negative likelihood ratio; DOR, } \\
\text { diagnose odds ratio. }\end{array}$ & & \\
\hline
\end{tabular}


Table 4

Performance of Combination of Serum Biomarkers in the Diagnosis of PJI

\begin{tabular}{|lllll|}
\hline & NLR + CRP & NLR +ESR & CRP + ESR & NLR + CRP + ESR \\
\hline AUC & 0.781 & 0.741 & 0.778 & 0.767 \\
\hline $95 \%$ Cl & $0.692-0.855$ & $0.648-0.820$ & $0.689-0.852$ & $0.676-0.843$ \\
\hline Standard error & 0.049 & 0.055 & 0.049 & 0.051 \\
\hline Z-statistic & 5.801 & 4.367 & 5.648 & 5.203 \\
\hline P Value & $<0.001$ & $<0.001$ & $<0.001$ & $<0.001$ \\
\hline Sensitivity (\%) & 56.25 & 53.13 & 56.25 & 56.25 \\
\hline Specificity (\%) & 89.61 & 89.61 & 90.91 & 90.91 \\
\hline PPV (\%) & 69.2 & 68.0 & 72.0 & 72.0 \\
\hline NPV (\%) & 83.1 & 82.1 & 83.3 & 83.3 \\
\hline +LR & 5.41 & 5.11 & 6.19 & 6.19 \\
\hline -LR & 0.49 & 0.52 & 0.48 & 0.48 \\
\hline \begin{tabular}{l} 
Accuracy (\%) \\
\hline DOR
\end{tabular} & 79.82 & 78.90 & 80.73 & 80.73 \\
\hline $\begin{array}{l}\text { PJI, periprosthetic joint infection; NLR, neutrophil-lymphocyte ratio; CRP, C-reactive protein; ESR, } \\
\text { erythrocyte sedimentation rate; AUC, area under curve; Cl, confidence interval; PPV, positive predictive } \\
\text { value; NPV, negative predictive value; +LR, positive likelihood ratio; -LR, negative likelihood ratio; DOR, } \\
\text { diagnose odds ratio. }\end{array}$ & & & \\
\hline
\end{tabular}

Table 5

Comparison of the Diagnostic Performance of Combination of Biomarkers in the Diagnosis of PJI

\begin{tabular}{|llc|}
\hline & AUC Difference & P Value \\
\hline NLR + CRP vs. NLR + ESR & 0.040 & 0.327 \\
\hline NLR + CRP vs. CRP + ESR & 0.003 & 0.928 \\
\hline NLR + ESR vs. CRP + ESR & 0.037 & 0.042 \\
\hline NLR + CRP vs. NLR + CRP + ESR & 0.014 & 0.656 \\
\hline NLR + ESR vs. NLR + CRP + ESR & 0.026 & 0.084 \\
\hline CRP + ESR vs. NLR + CRP + ESR & 0.011 & 0.060 \\
\hline $\begin{array}{l}\text { PJl, periprosthetic joint infection; NLR, neutrophil-lymphocyte ratio; CRP, C-reactive protein; ESR, } \\
\text { erythrocyte sedimentation rate; AUC, area under curve. }\end{array}$ & \\
\hline
\end{tabular}




\section{Discussion}

Despite the fact that the MSIS guideline has been recommending the use of CRP and ESR as useful biomarkers in aiding the diagnosis of PJI, some cases could still be missed during clinical practice, making the right diagnosis time-consuming and costly, and ultimately dramatically increasing the burden for patients. The investigation of markers that would improve the diagnosis is thus required. On this context, it should be noted that the association between preoperative levels of NLR and PJI is yet to be elucidated. In the present study, we investigated the diagnostic performance of the cost-effective and easily accessible NLR marker in facilitating the diagnosis of PJI, aiming to improve the timely and accurate detection of PJI. We found that the level of NLR was significantly higher in the PJI group than that in the aseptic mechanic failure group. However, the diagnostic value of NLR alone was demonstrated to not be a superior marker to those of CRP and ESR in diagnosing PJI, as shown in the analysis of the ROC curve, where its AUC and DOR values were lower than those of CRP and ESR. Nonetheless, when we combined NLR with CRP, this combination was shown to achieve nearly the same diagnostic value as the combination of CRP and ESR or the combination of all 3 markers together, enhancing the specificity and thus improving the ability to rule out PJI when used as a confirmatory test.

Nowadays, all patients who were admitted for different kinds of surgeries are required to have the CBC checked preoperatively, aiming to rule out contraindications of surgeries, such as systemic infection. Accordingly, NLR is calculated directly from the commonly ordered and readily available count of neutrophils and lymphocytes in CBC, making it easily accessible and cost-effective. Aside from that, NLR has been recognized as a marker of the systemic inflammatory response [8]. As such, NLR has been reported to reflect a heightened inflammatory reaction and has gained increasing attention as a prognostic marker in many conditions, such as solid tumors, inflammatory diseases, and postoperative infection $[10,11,14]$. A previous study reported that NLR was significantly higher in patients with polymyalgia rheumatica and was shown to be associated with disease activity and specific clinical features [10]. Inose et al. retrospectively investigated the association of NLR with the surgical site infection in 242 patients who underwent spinal instrumentation surgery. They found that the level of NLR at 6 to $7 \mathrm{~d}$ postoperatively was significantly related to the surgical site infection with a cutoff of 3.87 [14]. Several studies have explored the roles of NLR in cases of TJA. Yombi et al. investigated the distribution and fluctuation of NLR compared with that of CRP in 587 patients who underwent total knee arthroplasty, and found that NLR had a faster normalization and was more stable than CRP, demonstrating that NLR might be utilized as a potential biomarker to monitor postoperative inflammation or early infection [16]. Another study conducted by Golge found that NLR was significantly increased in patients with PJI [15]. Similarly, in our study, the level of NLR was significantly higher in patients with PJI in comparison with patients with aseptic mechanic failure, even after adjustments for age, sex, joint, diabetes mellitus, and hypertension, indicating that NLR might play a pivotal role in the inflammatory condition of patients with PJI.

Recently, more studies have been evaluating new biomarkers in diagnosing PJI, such as platelet count, mean platelet volume, fibrinogen, and NLR $[15,17,18]$. Our present study addressed that NLR had a fair 
diagnostic performance in predicting PJI with a sensitivity of $56.25 \%$, specificity of $80.52 \%$, and AUC of 0.658. In comparison to the study conducted by Golge [15], the sensitivity of NLR in our study was demonstrated to be relatively lower and the specificity higher when using an optimal cutoff of 2.52. Their study revealed that by using 2.45 as the optimal threshold, the sensitivity and specificity of NLR in detecting PJI was 90 and $72 \%$, respectively [15]. However, the study involved patients with either PJI or primary total knee arthroplasty, with the patients with primary arthroplasty being considered as the control group, which would have a great impact on the diagnostic performance of NLR because of the larger heterogeneity between the 2 groups. As the selection of patients is crucial for exploring the diagnostic value of markers, patients should be generalized under the consistent condition to decrease the confounding effect [19].

Further, combinations of biomarkers have been demonstrated to enhance diagnostic performance $[17,20$, 21]. Paziuk et al. demonstrated that the ratio of the platelet count to mean platelet volume was inferior to CRP and ESR when employed as a single index. However, when the ratio was used in combination with ESR and CRP, a statistically significant increase in the diagnostic performance of the combination of markers in predicting PJI was observed [17]. Qin et al. revealed that the combination of D-dimer and CRP improved the diagnostic performance in diagnosing PJI [21]. Likewise, we found that NLR alone was inferior to CRP and ESR, while when it was combined with CRP, this marker combination was demonstrated to achieve nearly the same diagnostic value as the combination of CRP and ESR or the combination of all 3 markers together, increasing the specificity and thus improving the ability to rule out PJI. Therefore, it was suggested that NLR, as a complementary biomarker with CRP, could improve the diagnostic performance in diagnosing PJI.

Many studies have investigated the role of CRP and ESR in diagnosing PJI, and have tried to establish an optimal threshold, as well as delineate the diagnostic performance of CRP and ESR for the diagnosis of PJI. Both the CRP and ESR, which are considered as specific indicators of infection, were shown to be highly elevated in the PJI group and to gradually decline postoperatively [20,22]. The sensitivity of CRP for diagnosing PJI has been reported to range from 74 to $94 \%$, whereas its specificity ranged from 20 to $100 \%$, with different predictive cutoffs [20,22-25]. On the other hand, the sensitivity and specificity of ESR were reported to vary from 42 to $94 \%$, and 33 to $90 \%$, respectively $[17,22-24,26]$. Compared with these reports, in our study, the sensitivity for CRP and ESR in predicting PJI was shown to be $62.50 \%$ and $59.38 \%$, while the specificity was $83.12 \%$ and $80.52 \%$, respectively. Except for the sensitivity of CRP, our obtained results were consistent with previous studies. The fluctuation observed in the sensitivity of CRP might be due to the variation of optimal thresholds and sample sizes yielded from different studies in comparison with our study $[19,23,26,27]$. A study by Bingham et al. tried to explain that different cutoff values would affect the diagnostic performance of a marker, and attempted to determine the optimal thresholds for ESR and CRP to achieve a sensitivity $\geq 95 \%$ compared with the optimal thresholds recommended by MSIS. They revealed that when $30 \mathrm{~mm} / \mathrm{h}$ and $10 \mathrm{mg} / \mathrm{L}$ were used as optimal thresholds for ESR and CRP, they observed an unacceptably low sensitivity and a high number of false negatives. To improve sensitivity and prevent any false negatives, an ESR and CRP threshold of $>10 \mathrm{~mm} / \mathrm{h}$ and $>$ $5 \mathrm{mg} / \mathrm{L}$, respectively was recommended to achieve a sensitivity $\geq 95 \%$ [27]. Obviously, different 
thresholds exert effect on diagnostic performance. Additionally, differences in the genetic background of different races have been reported to contribute to the discrepancy of the diagnostic performance of some markers, such as D-dimer [28].

There were several limitations associated with our study, which should be taken into account when interpreting our findings. First, this was a retrospective study conducted at a single medical center and therefore might have introduced selection bias. Not all confounding variables were considered into the analysis. Some of the confounding variables including body mass index, medications, underlying inflammatory or autoimmune conditions, specifically those that could impact the count of neutrophils or lymphocytes, were underestimated. Regarding the confounding factors we included, despite the significant differences reported for joint and hypertension status between the 2 groups, we used the multivariate logistic regression method to adjust the $\mathrm{p}$ value when comparing the aseptic and septic groups in order to subtract the confounding effects. Second, only patients requiring revision arthroplasty were enrolled in the study. Therefore, it was possible that patients with asymptomatic infections or mild clinical manifestations that did not need to undergo revision surgery were excluded from the study, predisposing it to a level of selection bias. Finally, the number of patients involved in our study, especially the PJI group, was relatively small. Recruiting more patients and better adjusting selection criteria in a future prospective study would allow for better representation.

\section{Conclusions}

In conclusion, our study demonstrated that the cost-effective and routinely obtained NLR biomarker was significantly higher in patients with PJI than in those with aseptic mechanical failure. When NLR was combined with CRP, the diagnostic value was improved, indicating NLR as a complementary marker in aiding the diagnosis of PJI. Patients exhibiting increasing levels of NLR should be closely monitored as this might indicate underlying inflammatory conditions and susceptibility to infection. However, more large-scale and prospective studies might be needed to further elucidate the diagnostic value of NLR in the diagnosis of PJI.

\section{Abbreviations}

TJA, total joint arthroplasty; PJI, periprosthetic joint infection; CRP, C-reactive protein; ESR, erythrocyte sedimentation rate; NLR, neutrophil-lymphocyte ratio; $C B C$, complete blood count; $R O C$, receiver operating characteristic; AUC, area under curve; PPV, positive predictive value; NPV, negative predictive value; +LR, positive likelihood ratio; -LR, negative likelihood ratio; DOR, diagnostic odds ratio; SD, standard deviation; RA, rheumatoid arthritis; AS, ankylosing spondyloarthritis; NEUT, neutrophil; LYM, lymphocyte; MSIS, musculoskeletal infection society; SPSS, statistical package for the social sciences.

\section{Declarations}

\section{Ethics approval and consent to participate}


The study was approved by the Institutional Review Board of First Affiliated Hospital of Sun Yat-sen University. The requirement for informed consent was waived due to the retrospective design of our study.

\section{Consent for publication}

Not applicable.

\section{Availability of data and materials}

The datasets used during the current study are available from the corresponding author on reasonable request.

\section{Competing interests}

There are no conflicts of interest to declare.

\section{Funding}

This work was supported by the China Postdoctoral Science Foundation [grant numbers: 2019M663272] and the National Natural Science Foundation of China [grant number: 81972050, 81672149].

\section{Authors' contributions}

YY, ZZ, PS contributed to the idea and design of the study. YY, MG, WC, GX, LZ, BP contributed to data collection, data analysis and drafted the manuscript. All authors contributed to the interpretation of results and manuscript revision. All authors read and approved the final manuscript.

\section{Acknowledgements}

We gratefully acknowledge the support of all doctors in our department.

\section{References}

1. Parvizi J, Tan TL, Goswami K, Higuera C, Della Valle C, Chen AF, et al. The 2018 definition of periprosthetic hip and knee infection: An evidence-based and validated criteria. J Arthroplasty. 2018;33:1309-14 e2.

2. Gwam CU, Mistry JB, Mohamed NS, Thomas M, Bigart KC, Mont MA, et al. Current epidemiology of revision total hip arthroplasty in the united states: National inpatient sample 2009 to 2013. J 
Arthroplasty. 2017;32:2088-92.

3. Kurtz SM, Lau E, Watson H, Schmier JK, and Parvizi J. Economic burden of periprosthetic joint infection in the united states. J Arthroplasty. 2012;27:61-5 e1.

4. Jamsen $E$, Varonen $M$, Huhtala $H$, Lehto MU, Lumio J, Konttinen $Y T$, et al. Incidence of prosthetic joint infections after primary knee arthroplasty. J Arthroplasty. 2010;25:87-92.

5. Huotari K, Peltola M, and Jamsen E. The incidence of late prosthetic joint infections: A registry-based study of 112,708 primary hip and knee replacements. Acta Orthop. 2015;86:321-5.

6. Amanatullah D, Dennis D, Oltra EG, Marcelino Gomes LS, Goodman SB, Hamlin B, et al. Hip and knee section, diagnosis, definitions: Proceedings of international consensus on orthopedic infections. $J$ Arthroplasty. 2019;34:S329-S37.

7. Perez-Prieto D, Portillo ME, Puig-Verdie L, Alier A, Martinez S, Sorli L, et al. C-reactive protein may misdiagnose prosthetic joint infections, particularly chronic and low-grade infections. Int Orthop. 2017;41:1315-9.

8. Mozos I, Malainer C, Horbanczuk J, Gug C, Stoian D, Luca CT, et al. Inflammatory markers for arterial stiffness in cardiovascular diseases. Front Immunol. 2017;8:1058.

9. Dolan RD, McSorley ST, Park JH, Watt DG, Roxburgh CS, Horgan PG, et al. The prognostic value of systemic inflammation in patients undergoing surgery for colon cancer: Comparison of composite ratios and cumulative scores. $\mathrm{Br} J$ Cancer. 2018;119:40-51.

10. Jung JY, Lee E, Suh $\mathrm{CH}$, and Kim HA. Neutrophil-to-lymphocyte ratio and platelet-to-lymphocyte ratio are associated with disease activity in polymyalgia rheumatica. J Clin Lab Anal. 2019:e23000.

11. Templeton AJ, Knox JJ, Lin X, Simantov R, Xie W, Lawrence N, et al. Change in neutrophil-tolymphocyte ratio in response to targeted therapy for metastatic renal cell carcinoma as a prognosticator and biomarker of efficacy. Eur Urol. 2016;70:358-64.

12. Canbolat N, Buget Ml, Sivrikoz N, Altun D, and Kucukay S. The relationship between neutrophil to lymphocyte ratio and postoperative pain in total knee and hip arthroplasty. Rev Bras Anestesiol. 2019;69:42-7.

13. Barker T, Rogers VE, Henriksen VT, Brown KB, Trawick RH, Momberger NG, et al. Is there a link between the neutrophil-to-lymphocyte ratio and venous thromboembolic events after knee arthroplasty? A pilot study. J Orthop Traumatol. 2015;17:163-8.

14. Inose H, Kobayashi Y, Yuasa M, Hirai T, Yoshii T, and Okawa A. Procalcitonin and neutrophil lymphocyte ratio after spinal instrumentation surgery. Spine (Phila Pa 1976). 2019;44:E1356-E61.

15. Golge UH. Neutrophil to lymphocyte ratio may be a diagnostic marker for prosthetic joint infection. $J$ Clin Anal Med. 2016;7: 218-21.

16. Yombi JC, Schwab PE, and Thienpont E. Neutrophil-to-lymphocyte ratio (NLR) distribution shows a better kinetic pattern than C-reactive protein distribution for the follow-up of early inflammation after total knee arthroplasty. Knee Surg Sports Traumatol Arthrosc. 2016;24:3287-92. 
17. Paziuk T, Rondon AJ, Goswami K, Tan TL, and Parvizi J. A novel adjunct indicator of periprosthetic joint infection: Platelet count and mean platelet volume. J Arthroplasty. 2019;35:836-9.

18. Li R, Shao HY, Hao LB, Yu BZ, Qu PF, Zhou YX, et al. Plasma fibrinogen exhibits better performance than plasma D-dimer in the diagnosis of periprosthetic joint infection: A multicenter retrospective study. J Bone Joint Surg Am. 2019;101:613-9.

19. Bernard L, Lubbeke A, Stern R, Bru JP, Feron JM, Peyramond D, et al. Value of preoperative investigations in diagnosing prosthetic joint infection: Retrospective cohort study and literature review. Scand J Infect Dis. 2004;36:410-6.

20. Glehr M, Friesenbichler J, Hofmann G, Bernhardt GA, Zacherl M, Avian A, et al. Novel biomarkers to detect infection in revision hip and knee arthroplasties. Clin Orthop Relat Res. 2013;471:2621-8.

21. Qin L, Li F, Gong X, Wang J, Huang W, and Hu N. Combined measurement of D-dimer and C-reactive protein levels: Highly accurate for diagnosing chronic periprosthetic joint infection. J Arthroplasty. 2020;35:229-34.

22. Xiong L, Li S, and Dai M. Comparison of D-dimer with CRP and ESR for diagnosis of periprosthetic joint infection. J Orthop Surg Res. 2019;14:240.

23. Xu H, Xie J, Huang Q, Lei Y, Zhang S, and Pei F. Plasma fibrin degradation product and D-dimer are of limited value for diagnosing periprosthetic joint infection. J Arthroplasty. 2019;34:2454-60.

24. Saleh A, George J, Faour M, Klika AK, and Higuera CA. Serum biomarkers in periprosthetic joint infections. Bone Joint Res. 2018;7:85-93.

25. Shahi A, Kheir MM, Tarabichi M, Hosseinzadeh HRS, Tan TL, and Parvizi J. Serum D-dimer test is promising for the diagnosis of periprosthetic joint infection and timing of reimplantation. $J$ Bone Joint Surg Am. 2017;99:1419-27.

26. Ghanem E, Antoci V, Jr., Pulido L, Joshi A, Hozack W, and Parvizi J. The use of receiver operating characteristics analysis in determining erythrocyte sedimentation rate and C-reactive protein levels in diagnosing periprosthetic infection prior to revision total hip arthroplasty. Int $\mathrm{J}$ Infect Dis. 2009;13:e444-9.

27. Bingham JS, Hassebrock JD, Christensen AL, Beauchamp CP, Clarke HD, and Spangehl MJ. Screening for periprosthetic joint infections with ESR and CRP: The ideal cutoffs. J Arthroplasty. 2019.

28. Zakai NA, McClure LA, Judd SE, Kissela B, Howard G, Safford M, et al. D-dimer and the risk of stroke and coronary heart disease. The reasons for geographic and racial differences in stroke (REGARDS) study. Thromb Haemost. 2017;117:618-24.

\section{Figures}


A

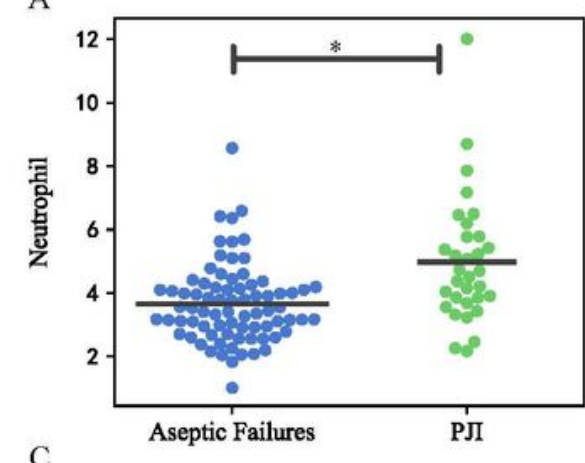

C

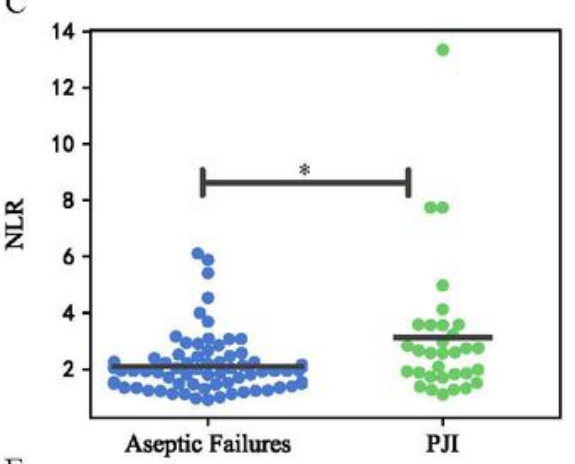

$\mathrm{E}$

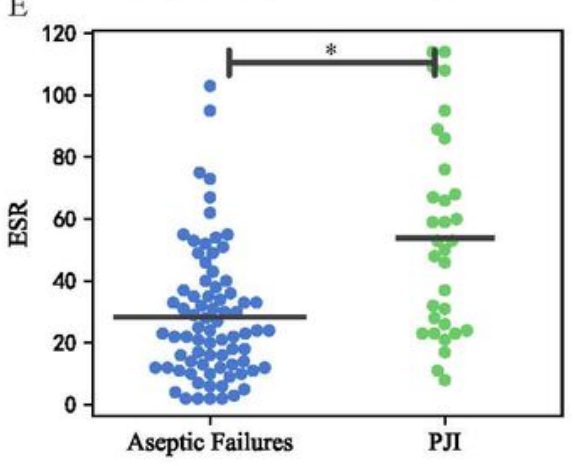

B

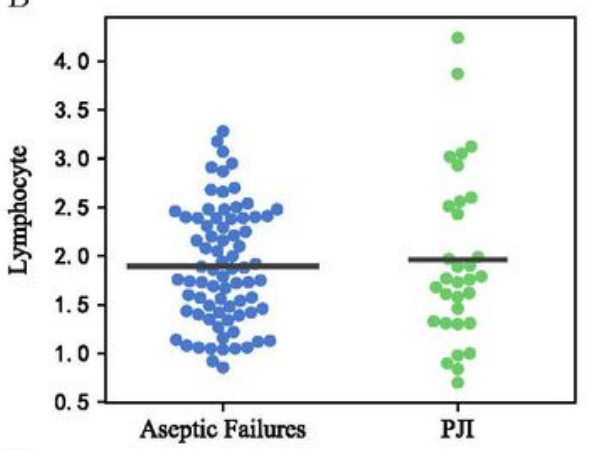

D

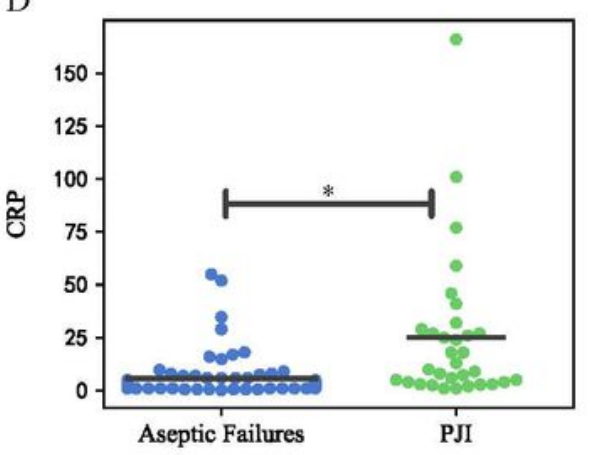

\section{Figure 1}

Comparisons of neutrophil, lymphocyte, NLR, CRP, and ESR between aseptic mechanic failure and PJI patients. A) Neutrophil, B) Lymphocyte, C) NLR, D) CRP, E) ESR. The asterisks indicate a significant difference between groups. The $P$ values were calculated by Mann-Whitney $U$ test. $P<0.05$ indicates a significant difference between groups. NLR, neutrophil-lymphocyte ratio; CRP, C-reactive protein; ESR, erythrocyte sedimentation rate. The black line represents the mean value of each group. 

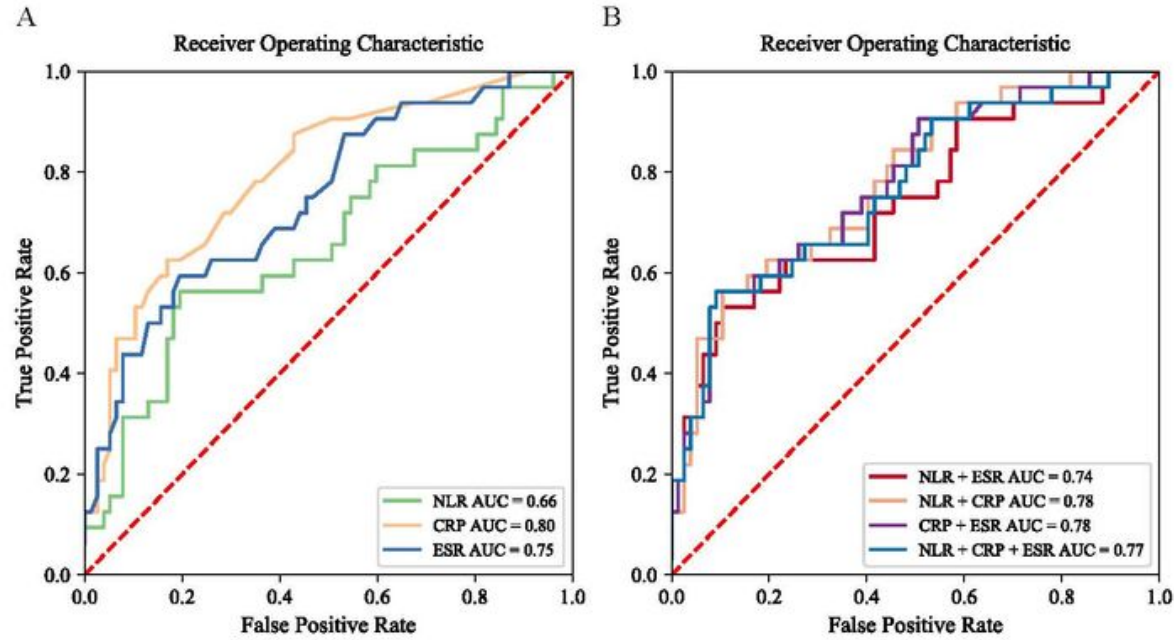

\section{Figure 2}

ROC curve of NLR, CRP, ESR, and combination to predict PJI. A) The diagnostic performance of each marker alone. B) The diagnostic performance of the combination of markers. NLR, neutrophil-lymphocyte ratio; CRP, C-reactive protein; ESR, erythrocyte sedimentation rate; AUC, area under curve; PJI, periprosthetic joint infection. 